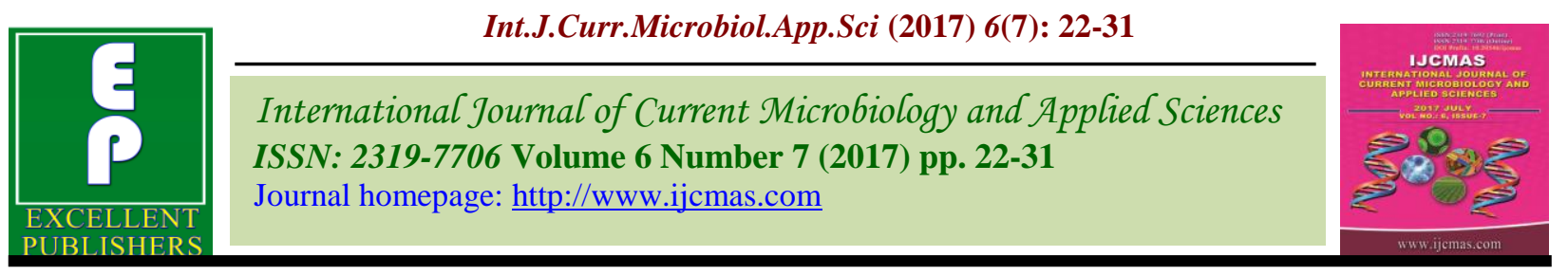

Original Research Article

https://doi.org/10.20546/ijcmas.2017.607.004

\title{
Antibiogram of Escherichia coli Isolates from Community Acquired Urinary Tract Infection: Special Reference to Fluoroquinolones Resistance
}

\author{
Neha Mangal*, Anamika Vyas, Mritunjay Kumar and A.S. Dalal \\ Department of Microbiology, Geetanjali Medical College and Hospital, Udaipur, India \\ *Corresponding author
}

\author{
A B S T R A C T
}

Keywords

Urinary Tract

Infection,

Antimicrobial

susceptibility,

Resistance,

Sensitivity,

Fosomysin,

Nitrofurantoin,

Ciprofloxacin,

Fluoroquinolone.

Article Info

Accepted:

04 June 2017

Available Online:

10 July 2017
Urinary tract infections are common bacterial infections that affect the urethra, bladder, ureter or the kidney. It is the second most common infectious presentation in community medical practice with a high rate of morbidity and financial cost. This infection has burden rate of 150 million cases are estimated per annum while around 8 million cases are attributed to UTI in the USA. The study was conducted in the department of Microbiology, GMCH, Udaipur (Rajasthan), India during the period of year 2014-2015 on 356 patients, clinically diagnosed as Community acquired UTI. 100 non-duplicate urinary isolates of Escherichia coli from patients with clinically evident UTI were included in the study. Samples were collected from the patients after obtaining an informed verbal consent. Method employed for collection of urine was Midstream clean catch technique. Institutional Ethical clearance was obtained before commencement of the study. All relevant laboratory records of every subject was systemically recorded in pre designed data sheet. In this study, age group of 46-55 years was predominantly affected, irrespective of gender. Out of 100 screened strains of Escherichia coli, 33 were found to be ESBL and 67 to be non EBBL producers. The most resistant drug in Urinary Pathogenic Escherichia coli (UPEC) isolates was Ciprofloxacin with resistance rate of $82 \%$. No resistance was observed for Fosomysin. Nitrofurantoin was found to be most susceptible drug with sensitivity rate of 98\%. Fosomysin and Nitrofurantoin was found to retain 100\% sensitivity, against non ESBL producers. Out of 82 Ciprofloxacin-resistant isolates, Nalidixic acid was most resistant drug with $100 \%$ resistance rate. Conclusion: Both Fosomysin and Nitrofurantoin can be used as drug of choice for empirical treatment of community acquired UTI. Resistance to Ciprofloxacin should not be considered as marker for resistance to other Fluoroquinolone. Thus Fluoroquinolone should only be prescribed following culture and sensitivity testing but not for empirical therapy.

\section{Introduction}

Urinary Tract Infection is the second most common infectious presentation in community medical practice (Kyung-Hwa et al., 2014). It is one of the most commonly occurring medical problems, causing considerable morbidity and healthcare costs (Smita Sood et al., 2012).
Urinary tract infection (UTI), considered among the most common bacterial diseases that affect a large part of the world's population. Urinary tract infection is generally treated empirically by general practitioners, for which they need to be aware of the locally prevalent strains and their 
sensitivity pattern. Over the last few decades the resistance pattern of urinary isolates has been showing dramatic changes all over the world (Kadri et al., 2004).

In the United States, UTI account for approximately seven million office visits to physicians each year and over one million hospitalizations annually are attributed to or complicated by UTI (Orenstein et al., 1999).

UTI is an inflammatory response of the ureothelium to bacterial invasion that is usually associated with bacteriuria and pyuria (Campbell-Walsh Urology $10^{\text {th }}$ edition).

UTI is a broad term that describes microbial colonization of the urine and infection of the structures of the urinary tract - kidney, renal pelvis, ureters, bladder, and urethra, as well as adjacent structures such as the perinephric fascia, prostate, and epididymis (Kunin, 1997). UTI may be asymptomatic (subclinical infection) or symptomatic (disease). Thus, the term UTI encompasses a variety of clinical entities, including asymptomatic bacteriuria (ABU), cystitis, prostatitis and pyelonephritis (Harrison's Principles of Internal Medicine).

When chemotherapeutic agents used to treat UTI were introduced in the early twentieth century, they were relatively ineffective, and persistence of infection after 3 weeks of therapy was common. Nitrofurantoin, which became available in the 1950s, was the first tolerable and effective agent for the treatment of UTI. This study aims to find out antibiogram of Escherichia coli isolates from Community Acquired Urinary Tract Infection with special reference to fluoroquinolones resistance.

\section{Materials and Methods}

The study was conducted in the department of Microbiology, GMCH, Udaipur (Rajasthan), India during the period of 2014-2015.
100 non-duplicate urinary isolates of Escherichia coli from patients with clinically evident UTI were included in the study. Samples were collected from the patients after obtaining an informed verbal consent. Method employed for collection of urine was Midstream "clean catch" technique. Institutional Ethical clearance was obtained before commencement of the study. All relevant laboratory records of every subject was systemically recorded in pre designed data sheet.

\section{Inclusion criteria}

Outdoor patients of age 15-50 years of both the sexes with symptomatic UTI.

In patients who had UTI at the time of admission.

\section{Exclusion criteria}

Patient with history of hospital admission a week before their presentation in OPDs.

Patient with indwelling catheterization.

Patient on antibiotic therapy in past 5 days will be excluded.

Patient who is not willing to participate.

\section{Collection of urine sample}

The clean catch mid-stream technique was employed to collect urine samples.

\section{For female patients}

After proper positioning of thigh, patient was instructed to spread the labia with one hand and clean the periurethral area and the perineum with 2-3 gauze pads saturated with soapy water, using a forward-to-back motion, followed by a rinse with sterile saline or water. Then pass a small amount of urine into 
toilet, and finally urinate into the wide mouthed container.

\section{For male patient}

After washing his hand, clean catch midstream urine will be collected with foreskin separated.

Urine sample was collected aseptically in a sterile wide mouthed container. Each sample was properly labeled with patient name, OPD number etc. the specimens were then transferred to the laboratory as quickly as possible, usually within 1 hour after collection.

The sample was inoculated on Sheep blood agar and MacConkey agar (Hi Media Laboratories, Mumbai, India). The inoculated plates were incubated aerobically at $37^{\circ} \mathrm{c}$ for 24 hours and 48 hours for negative cases. After completion of incubation, the inoculated culture plates were observed for the presence of any bacterial growth. Colony count was done to calculate the number of colony forming unit per $\mathrm{ml}$ of urine.

The urine cultures which yielded significant bacteriuria $\left(>10^{5} \mathrm{cfu} / \mathrm{ml}\right)$ was included for further analysis. Plates were observed for typical colony characteristics and organisms were identified on basis of their cultural and biochemical properties.

\section{Results and Discussion}

A total of 356 patients, clinically diagnosed as Community acquired UTI, were included in study. Bacterial culture of urine specimen, of these patients was performed. Among these 356 , bacterial growth were $151(42.42 \%)$, $201(56.46 \%)$ had no growth and $4(1.12 \%)$ had mixed growth (Table 1). The culture positivity of UTI among the female were $(52 \%)$ in Community acquired UTI. Culture positivity among the male were (48\%). The predominant age group in case of Community acquired UTI was 46-55 (Table 2).

Table 3 shows the aerobic organism that was isolated in culture. Out of 151 isolates the overall prevalence of $E$. coli was found $66.23 \%(\mathrm{n}=100)$ in positive sample of urine. Escherichia coli were the most prevalent organism. It was followed by Coagulase negative Staphylococcus $10.6 \% \quad(\mathrm{n}=16)$, Enterococcus $9.9 \% \quad(\mathrm{n}=15)$, Pseudomonas aeruginosa $4.63 \% \quad(\mathrm{n}=7)$, Klebsiella $4 \%$ $(\mathrm{n}=6)$, Candida species $1.99 \%(\mathrm{n}=3)$, Proteus mirabilis $1.99 \%(\mathrm{n}=3)$ and Coagulase positive Staphylococcus $0.66 \%(\mathrm{n}=1)$.

Out of 100 strains of Escherichia coli 33 strains were Extended Beta Lactamase Producer (ESBL Producer) and 67 were nonESBL producers. It was detected in accordance to guideline of CLSI. It is a confirmatory test, done by using Ceftazidime (30mcg) and Ceftazidime/Clavulanic acid (30/10mcg) discs.

The most resistant drug in UPEC isolates was Ciprofloxacin with resistance rate of $(82 \%)$ followed by Ampicillin (81\%), Amoxycillin/ Clavulanic acid (78\%), Cefazolin (77\%), Cefuroxime (76\%), Cefotaxime (76\%), Ceftazidime (66\%) and Cefixime (76\%). CoTrimoxazole resistance rate was found to be (63\%). Piperacillin/Tazobactam, Meropenam, Amikacin Nitrofurantoin was found to be susceptible drug with sensitivity rate of $87 \%$, $90 \%, 91 \%$ and $98 \%$ respectively. Fosomycin has maximum sensitivity rate of $100 \%$ (Table 4).

The most resistant drug in ESBL producing Escherichia coli isolates was found Ampicillin (100\%), Amoxycillin/Clavulanic acid $(100 \%)$, Cefazolin (100\%), Cefuroxime (100\%), Cefotaxime (76\%), Ceftazidime $(100 \%)$ and Cefixime (100\%). Ciprofloxacin 
ressistance rate was found to be $(100 \%)$. CoTrimoxazole resistance rate was found to be (72.72\%). Nitrofurantoin was found to be susceptible drug with $93.93 \%$ sensitivity rate. Fosomycin, Piperacillin/ Tazobactam, Meropenam, Amikacin all has maximum sensitivity rate of $100 \%$ to ESBL producing Escherichia coli (Table 4).

The most resistant drug in non-ESBL producing Escherichia coli isolates was Ciprofloxacin with resistance rate of $(73.13 \%)$. Resistance rate of Ampicillin was (71.64\%), Amoxycillin/Clavulanic acid (67.16\%), Cefazolin (65.67\%), Cefuroxime (64.18\%), Cefotaxime (64.18\%), Cefixime $(64.18 \%)$ and Ceftazidime (49.25\%). CoTrimoxazole resistance rate was found to be (58.2\%). Amikacin, Meropenam, Piperacillin/Tazobactam was found to be susceptible drug with $86.57 \%, 85.1 \%$ and $80.6 \%$ sensitivity rate respectively. Fosomycin and Nitrofurantoin was highly sensitive antimicrobial agent with sensitivity rate of $100 \%$ to non-ESBL producing Escherichia coli (Table 4).

Nalidixic acid is the most resistant drug, with resistant rate of $100 \%$. Resistant rate of Prulifloxacin was $65.85 \%$, Ofloxacin $68.29 \%$, Levofloxacin $19.5 \%$ while that of Norfloxacin was only $8.53 \%$ (Table 5).

In the present study, 356 urine samples from clinical cases of community acquired UTI was processed, out of which 151 (42.42\%) were culture positive. Four (1.12\%) samples yielded growth of more than one bacterial isolate. No growth was observed in 201 $(56.46 \%)$ samples. Similar to our findings, Biswas et al., (2014) had reported $42.45 \%$ culture positivity from urine samples. In comparison to our yield; lower positivity rate of $36.68 \%$ and $38 \%$ was reported by Mehta et al., (2013) and Ritu et al., (2009) respectively. In contrast to our findings, higher culture positivity rate of $65 \%$ in community acquired UTI was reported by Indu Sharma et al.

This variation may be attributed to the media selection, technique of growth and local prevalence rate. Even the standard personal hygiene and education status may be responsible.

In the present study, $62 \%$ of culture positive samples were from female and $38 \%$ were from males. In studies carried by Smita Sood et al., (2012) and Devanad Prakash et al., (2013); both had reported higher prevalence of UTI in females $62.42 \%$ and $65 \%$ respectively, whereas $37.67 \%$ and $35 \%$ in males. It was mainly due to anatomic and physiological factors.

In the current study $42 \%$ subjects were in between the age group of 46-55 years, which is similar to the finding of Amarjit Singh Vij et al., and Devanand Prakash et al., (2013); followed by $26 \%$ subjects in between $36-45$ years and $16 \%$ of 15-25 years age group. In contrast to our findings, studies conducted by Dash et al., (2013) and Razak et al., (2012) showed maximum incidence of UTI among younger patients of age group 20-40 years. This variation may be due to the geographical and demographics of the region.

In our findings, Gram-negative uropathogens constituted the major part of the isolates 76.82\% (116/151). Gram-positive uropathogens constituted $23.18 \%(35 / 151)$ of the total isolates. Out of $76.82 \%$ of Gram negative isolates, Escherichia coli was contributed by (66.23\%); Pseudomonas aeruginosa (4.63\%), Klebsiella species (4\%); Proteus mirabilis (1.99\%). Among $23.18 \%$ of gram positive, Coagulase Negative Staphylococci species was contributed by (10.6\%), Enterococcus species (9.9\%) and Coagulase Positive Staphylococci (0.66\%). 
Among yeast isolates (1.99\%) Candida species was isolated. Escherichia coli $66.23 \%$ (100/151) was found the most prevalent gram negative bacteria in the positive urine samples of UTI. Similarly, to our findings Amarjit Singh Vij et al., (2013) reported with E. coli prevalence rate of $68.7 \%$ and Moges Tiruneh et al., (2012) reported rate of 42.3\%, Gupta et al., (2001) in USA found $75 \%$ E. coli. A study done by Dyer et al., (1998) showed that the proposition of $E$. coli in the current decade has risen significantly, it accounted for $69 \%$ of positive cultures in 1991, which increased to $75 \%$ in 1994 and $81 \%$ in 1997.

Table.1 Result of growth in culture

\begin{tabular}{|c|c|c|c|}
\hline \multirow{2}{*}{ Growth result } & \multicolumn{3}{|c|}{ Number of strains isolated } \\
\cline { 2 - 4 } & Male (\%) & Female (\%) & Total (\%) \\
\hline Bacterial growth & $72(39.78 \%)$ & $79(45.14 \%)$ & $151(42.22 \%)$ \\
\hline No growth & $108(59.67 \%)$ & $93(53.14 \%)$ & $201(56.46 \%)$ \\
\hline Mixed growth & $1(0.55 \%)$ & $3(1.72 \%)$ & $04(1.12 \%)$ \\
\hline Total & $\mathbf{1 8 1 ( 1 0 0 \% )}$ & $\mathbf{1 7 5}(\mathbf{1 0 0 \%})$ & $\mathbf{3 5 6}(\mathbf{1 0 0 \% )})$ \\
& $\mathbf{( 5 0 . 8 4 \% )}$ & $\mathbf{( 4 9 . 1 6 \% )}$ & $\mathbf{( 1 0 0 \% )}$ \\
\hline
\end{tabular}

Table.2 Age and sex distribution of the culture positive urine samples

\begin{tabular}{|c|c|c|c|}
\hline Age (years) & Male (\%) & Female (\%) & Total (\%) \\
\hline $15-25$ & $10(13.89 \%)$ & $14(17.72 \%)$ & $24(15.89 \%)$ \\
\hline $26-35$ & $12(16.67 \%)$ & $13(16.46 \%)$ & $25(16.56 \%)$ \\
\hline $36-45$ & $16(22.22 \%)$ & $22(27.85 \%)$ & $38(25.16 \%)$ \\
\hline $46-55$ & $34(47.22 \%)$ & $30(37.97 \%)$ & $64(42.38 \%)$ \\
\hline \multirow{2}{*}{ Total } & $\mathbf{7 2 ( 1 0 0 \% )}$ & $\mathbf{7 9 ( 1 0 0 \% )}$ & $\mathbf{1 5 1 ( 1 0 0 \% )}$ \\
& $\mathbf{( 4 7 . 6 9 \% )}$ & $\mathbf{( 5 2 . 3 2 \% )}$ & $(\mathbf{1 0 0 \% )}$ \\
\hline
\end{tabular}

Table.3 Bacteriological profile of urine specimen

\begin{tabular}{|c|c|c|c|}
\hline \multirow{2}{*}{ ORGANISM ISOLATED } & \multicolumn{3}{|c|}{ Number of isolates } \\
\hline & Male (\%) & Female (\%) & Total $(\%)$ \\
\hline Escherichia coli & $47(65.28 \%)$ & $53(67.09 \%)$ & $100(66.23 \%)$ \\
\hline $\begin{array}{l}\text { Coagulase Negative } \\
\text { Staphylococci species }\end{array}$ & $7(9.72 \%)$ & $9(11.39 \%)$ & $16(10.6 \%)$ \\
\hline Enterococcus species & $7(9.72 \%)$ & $8(10.13 \%)$ & $15(9.9 \%)$ \\
\hline Pseudomonas aeruginosa & $4(5.56 \%)$ & $3(3.80 \%)$ & $07(4.63 \%)$ \\
\hline Klebsiella species & $4(5.56 \%)$ & $2(2.53 \%)$ & $06(4.00 \%)$ \\
\hline Candida species & $2(2.78 \%)$ & $1(1.27 \%)$ & $03(1.99 \%)$ \\
\hline Proteus mirabilis & $1(1.39 \%)$ & $2(2.53 \%)$ & $03(1.99 \%)$ \\
\hline $\begin{array}{c}\text { Coagulase Positive } \\
\text { Staphylococci } \text { species }\end{array}$ & Nil & $1(1.27 \%)$ & $01(0.66 \%)$ \\
\hline TOTAL & $\begin{array}{l}72(100 \%) \\
(47.69 \%)\end{array}$ & $\begin{array}{l}79(100 \%) \\
(52.32 \%)\end{array}$ & $\begin{array}{c}151(100 \%) \\
(100 \%)\end{array}$ \\
\hline
\end{tabular}


Table.4 Antibiotic susceptibility of ESBL and non-ESBL Escherichia coli producers

\begin{tabular}{|c|c|c|c|c|c|c|}
\hline \multirow[t]{2}{*}{ Antibiotic } & \multicolumn{2}{|c|}{$\begin{array}{l}\text { ESBL producers } \\
\quad(\mathbf{n}=\mathbf{3 3})\end{array}$} & \multicolumn{2}{|c|}{$\begin{array}{l}\text { Non-ESBL Producers } \\
\qquad(\mathrm{n}=67)\end{array}$} & \multicolumn{2}{|c|}{$\begin{array}{l}\text { Escherichia coli } \\
\text { Producers } \\
(\mathbf{n}=100)\end{array}$} \\
\hline & Resistant & Sensitive & Resistant & Sensitive & Resistant & Sensitive \\
\hline Ampicillin & $33(100 \%)$ & Nil & $48(71.64 \%)$ & $19(28.36 \%)$ & 81 & 19 \\
\hline Amikacin & Nil & $33(100 \%)$ & $9(13.43 \%)$ & $58(86.57 \%)$ & 9 & 91 \\
\hline $\begin{array}{l}\text { Amoxycillin/Cla } \\
\text { vulanic acid }\end{array}$ & $33(100 \%)$ & Nil & $45(67.16 \%)$ & $22(32.84 \%)$ & 78 & 22 \\
\hline Co-Trimoxazole & $24(72.73 \%)$ & $9(27.27 \%)$ & $39(58.21 \%)$ & $28(41.79 \%)$ & 63 & 37 \\
\hline Cefazolin & 33 & Nil & $44(65.67 \%)$ & $23(34.33 \%)$ & 77 & 23 \\
\hline Cefuroxime & $33(100 \%)$ & Nil & $43(64.18 \%)$ & $24(35.82 \%)$ & 76 & 24 \\
\hline Cefixime & $33(100 \%)$ & Nil & $43(64.18 \%)$ & $24(35.82 \%)$ & 76 & 24 \\
\hline Cefotaxime & $33(100 \%)$ & Nil & $43(64.18 \%)$ & $24(35.82 \%)$ & 76 & 24 \\
\hline Ceftazidime & $33(100 \%)$ & Nil & $33(49.25 \%)$ & $34(50.75 \%)$ & 66 & 34 \\
\hline Fosfomycin & Nil & $33(100 \%)$ & Nil & $67(100.0 \%)$ & 0 & 100 \\
\hline $\begin{array}{l}\text { Piperacillin/Tazo } \\
\text { bactam }\end{array}$ & Nil & $33(100 \%)$ & $13(19.40 \%)$ & $54(80.60 \%)$ & 13 & 87 \\
\hline Nitrofurantoin & $2(6.06 \%)$ & $31(93.94 \%)$ & Nil & $67(100.05)$ & 2 & 98 \\
\hline Meropenem & Nil & $33(100 \%)$ & $10(14.93 \%)$ & $57(85.07 \%)$ & 10 & 90 \\
\hline Ciprofloxacin & $33(100 \%)$ & Nil & $49(73.13 \%)$ & $18(26.875)$ & 82 & 18 \\
\hline
\end{tabular}

Table.5 Fluoroquinolones susceptibilities for 82 ciprofloxacin resistant Escherichia coli

\begin{tabular}{|c|c|c|}
\hline ANTIBIOTIC & RESISTANT & SENSITIVE \\
\hline Nalidixic acid & 82 & 0 \\
\hline Norfloxacin & 7 & 75 \\
\hline Ofloxacin & 56 & 26 \\
\hline Levofloxacin & 16 & 66 \\
\hline Prulifloxacin & 54 & 28 \\
\hline
\end{tabular}

E. coli had been the predominant organism isolated and no significant change has occurred in this picture over the last couple of decades. The reason of highest rate of isolation of E. coli causing UTI is due to the fact that most of the bacterial organisms causing UTI originate from the faecal flora and among these facultative anaerobes, E. coli constitutes the major portion superimposed by various virulence factors that facilitate the ascent of bacteria from faecal flora, introitus or periurethral area, up the urethra into the bladder and less frequently allow the organisms to reach the kidneys to induce symptomatic inflammation (Dyer et al.,
1997). In present study, the antibiogram of UPEC isolates were done using Clinical and Laboratory Standards Institute guidelines (CLSI 2012). Among 100 isolates of UPEC; the resistance pattern which was observed for Ciprofloxacin (82\%), Ampicillin (81\%), Amoxycillin/Clavulanic acid (78\%), Cefazolin (77\%), Cefuroxime (76\%), Cefixime (76\%), Cefotaxime (76\%), Ceftazidime (66\%), Co-Trimoxazole (63\%), Piperacillin/Tazobactam (13\%), Meropenam (10\%), Amikacin (9\%), Nitrofurantoin (2\%), Fosfomycin (0\%). Fosfomycin was also included in this study as it is recommended by IDSA guidelines for optimal treatment of CA- 
UTI. No resistance was observed for this antimicrobial agent. In our study, isolates with higher resistance were observed towards Ampicillin (81\%), Amoxycillin + Clavulinic acid $(78 \%)$. Same result was found in study done by Smita Sood et al., (2012), in which resistance rate to Ampicillin and Amoxycillin+Clavulinic acid were recorded $(81.18 \%)$ and $(80.69 \%)$ respectively. Our result correlates with a study done by Biswas et al., (2014), in which resistance rate to Amoxycillin+Clavulinic acid was (84.17\%). UPEC showed higher resistance to older urinary microbial agents, which indicates that increased consumption of a particular antibiotic, can be a pathway to its resistance. Findings of this study, thus suggest that empirical treatment with these drugs may lead to failure of treatment.

In present study, the resistance to trimethoprim-sulfamethoxazole was observed in $63 \%$ of isolates. Similarly to our findings Biswas et al., (2014), Hena Rani et al., (2011), reported resistance rate of $65 \%$ and $69 \%$ respectively. Our results were higher as compared to a study of Arsian et al., (2005), where trimethoprim/sulfamethoxazole resistance was observed in $36 \%$ of the uncomplicated UTIs. Trimethoprim/ sulfamethoxazole was the recommended treatment of choice for uncomplicated UTI according to the Infectious Diseases Society of America (IDSA) guidelines 2010. The IDSA also recommended that if resistance to Trimethoprim/sulfamethoxazole is greater than $20 \%$, it should not be considered for first-line empirical therapy, thus in our setting trimethoprim/sulfamethoxazole may not be recommended for empirical therapy in uncomplicated UTI's.

In our study resistance rate to meropenem was 10\%. Carbapenems (Meropenem) used in our study was found to be the sensitive drug against the isolates. The sensitivity rate of carbapenems was $90 \%$. Similar result was found with Mandal et al., (2012) with resistance rate of $9.2 \%$ and Devanad Prakash et al., (2015) with resistance rate of $4.55 \%$. Carbapenems are drug of choice for ESBL producers, as these strains are frequently multidrug resistant. Due to their high cost, parentral administration and the emergence of metallobetalactamase, it should not be used in community acquired UTI. Thus it should be restricted for use in critical patients where admission is required.

Fosfomycin is another oral antibiotic which is commonly used for treatment of CA-UTI. In our study susceptibility to fosfomycin was $100 \%$. Our result matches the study done by Asfia Sultan et al., (2015) in Aligarh Muslim University, Aligarh. Fosfomycin is an age-old drug. Resistance rate to this antimicrobial drug is low and most frequently acquired by chromosomal mutations that do not spread easily. The other benefits of use of fosfomycin are its less cost, dosage friendly, and non-toxic, nonallergic.

Fluoroquinolones are preferred as initial agents for empiric therapy of UTI, especially in those areas where resistance to first line of agent (Cotrimoxazol and Aminopenicillins) is likely to be concern. This is because they have high bacteriological and clinical cure rates. The resistance rate of ciprofloxacin was $82 \%$ in our study. The result of this study correlates with Deshmukh et al., (2014), Biswas et al., (2014) and Smita Sood et al., (2012) who reported resistance of $83.01 \%$, $90 \%$ and $75 \%$ respectively. Our result was higher than study conducted by John David Ohieku et al., (2013) in which the resistance rate for ciprofloxacin was only $42 \%$. Fluoroquinolones are a relatively new class of synthetic antibiotics with potent bactericidal, broad spectrum activity against many clinically important pathogens which are responsible for variety of infections and thus 
widely used. In past few years, fluoroquinolones have been prescribed more frequently for the treatment of UTI. This may have led to an increase in fluoroquinolonesresistant $E$. coli infections, which are difficult to treat (Goettsch et al.,). In present study Ciprofloxacin resistant strains were evaluated for other quinolones also.

Nalidixic acid is first generation fluoroquinolones. Maximum resistance was observed in it, with resistant rate of $100 \%$. Nalidixic acid was the first clinically available quinolone. However, because of its pharmacokinetic properties and the emergence of resistant isolates, its clinical use has been abandoned. Another mechanism can be as this is an older urinary microbial agent, which indicates that increased consumption of this antibiotic can be a pathway to its resistance.

Among the fluoroquinolones; Ofloxacin, levofloxacin, norfloxacin and prulifloxacin all have excellent bioavailability and have higher urinary concentrations. Their penetration into prostatic and renal tissue is also excellent. In our study resistance towards Ofloxacin was $68.29 \%$, Prulifloxacin was $65.85 \%$, and Levofloxacin was $19.5 \%$; while that of Norfloxacin was only $8.53 \%$.

Similar finding was observed in the study of Sumera Sabir et al., (2014) where resistance to ciprofloxacin was $(54.2 \%)$ and that of norfloxacin (11.2\%). High resistance was observed in Ofloxacin due to its same mechanism of action and same generation as that of Ciprofloxacin. Prulifloxacin was not active against most of ESBL-producing gramnegative bacilli. This observation was similar to the previous reports from other studies (Giannarini et al., 2009). Levofloxacin achieve very high urine concentrations for eradication of most of the organisms that cause UTIs. Among recently developed fluoroquinolones, levofloxacin is widely used in clinical practice because of its established efficacy and safety (Wimer, 1998; Norby et al., 1998). Levofloxacin is less likely to select resistant strains compared with older quinolone (Drugeon, 1999). In our study, not much resistance was noted against Norfloxacin; it is due to the less use of this drug in past few years.

The increased prevalence of antimicrobial resistance among UPEC limits the therapeutic options considerably. Both Fosomysin and Nitrofurantoin can be used as drug of choice for empirical treatment of community acquired UTI. Resistance to Ciprofloxacin should not be considering as marker for resistance to other fluoroquinolone as ciprofloxacin- resistant isolates were sensitive to levofloxacin and norfloxacin. This increase in resistance of fluoroquinolone among urinary isolates of Escherichia coli, suggests that empirical therapy with fluoroquinolone often fails in patients with community UTI. This restricts its use as an empirical drug of choice in community acquired UTIs. Thus fluoroquinolone should only be prescribed following culture and sensitivity testing but not for empirical therapy.

\section{References}

Amarjit Singh Vij, Shashi Chopra, et al. Two years retrospective study of antibiotic resistance pattern of uropathogens especially Escherichia coli in north India. CIB Tech. J. Microbiol., ISSN: 2319-3867.

Andriole, V.T. 1998. The quinolones: Academic Press, New York.

Arsian, H., Azap, O.K., Ergonul, O., Timurkaynak, F. 2005. Risk factors for ciprofloxacin resistance among Escherichia coli strains isolated from community-acquired urinary tract 
infections in Turkey. J. Antimicrob. Chemother., 56(5): 914-8.

Asfia Sultan, Meher Rizvi, et al. 2015. Increasing antimicrobial resistance among uropathogens: Is fosfomycin the answer? Urol. Annals, Vol 7 Issue.

Biswas, N.K., Patel, P.H., Ahir, H.R., Kothadia, Nerurkar, A.B. 2014. Bacteriological profile of urinary tract infection and its antibiotic susceptibility at tertiary care hospital, Valsad, Gujarat, India. J. Pharm. Biomed. Sci., 04(09): 751-754.

Campbell-Walsh Urology $10^{\text {th }}$ edition.

Clinical and Laboratory Standards Institute (CLSI), 2007. Interpretive chart of zone sizes Performance Standards for Antimicrobial Susceptibility Testing.

Dash, M., Padhi, S., Mohanty, I., Panda, P. and Parida, B. 2013. Antimicrobial resistance in pathogens causing urinary tract infections in a rural community of Odisha, India. J. Family and Community Med., 20(1): 20-26.

Deshmukh, P.M. and Ukesh, C.S. Mutidrug. 2014. Resistance pattern in Escherichia coli isolated from urinary tract infected (UTI's) patients. Int. J. Life Sci., 2(1): 53-57.

Drugeon, H.B., Juvin, M.E. and Bryskier, A. 1999. Relative potential for selection of fluoroquinolone-resistant Streptococcus pneumoniae strains by levofloxacin: comparison with ciprofloxacin, sparfloxacin and ofloxacin. $J$. Antimicrobial Chemother., 43, Suppl. C, 55-9.

Dyer, I.E., Sankay, T.M. and Dawson, J.A. 1998. Antibiotic resistance in bacterial urinary tract infection 1991 to 1997. The West J. Med., 169: 265-268.

Giannarini, G., Tascini, C., Selli, C. 2009. Prulifloxacin: clinical studies of a broad-spectrum quinolone agent. Future Microbiol., 4: 13-24.
Goettsch, W., Van Pelt, W., Nagelkerke, N., et al. 2000. Increasing resistance to fluoroquinolones in Escherichia coli from urinary tract infections in The Netherlands. J. Antimicrobial Chemother., 46(2): 223-228.

Gupta, K., Hooton, T.M. and Stamm, W.E. 2001. Increasing antimicrobial resistance and the management of uncomplicated community acquired UTIs. Annals of Internal Med., 135: 4150.

Harrison's Principles of Internal Medicine, $18^{\text {th }}$ edition.

Indu Sharma, Paul, D., Prevalence of community-acquired urinary tract infections in Silchar Medical College, Assam, India and its antimicrobial susceptibility profile. IJMS, 66(11) and 12.

Kadri, S.M., Gash, B. and Rukhsana, A. Antibiotic Sensitivity and Resistance Profile of the Micro-organisms Responsible for Urinary Tract Infection Observed in Kashmir, India., Indian J. Practising Doctor, 1(1): 79-84.

Kunin, C.M. 1997. Urinary Tract Infections. Detection, Prevention, and Management. Williams and Wilkins, $5^{\text {th }}$ edition.

Kyung-Hwa Park, A., Won Sup Oh, B., Eu Suk Kim, C., Sang Won Park, et al. 2014. Factors associated with ciprofloxacin- and cefotaxime-resistant Escherichia coli in women with acute pyelonephritis in the emergency department. Int. J. Infect. Dis., 23: 8-13.

Mandal, J., Acharya, N.S., Buddhapriya, D. and Parija, S.C. 2012. Antibiotic resistance pattern among common bacterial uropathogens with a special reference to ciprofloxacin resistant Escherichia coli. Indian J. Med. Res., 136: 842-849.

Mehta, M., Bhardwaj, S. and Sharma, J. 2013. Screening of urinary isolates for the 
prevalence and antimicrobial susceptibility of Enterobacteria other than Escherichia coli. Int. J. Life Sci. Pharma Res., 3(1): 100-104.

Moges Tiruneh, Sisay Yifru, et al. 2014. Changing trends in prevalence and antibiotics resistance of uropathgens in patients attending the Gondar University Hospital, Northwest Ethopia. $I J B$, article ID 629424.

Norby, S.R., Petermann, W., Willcox, P.A., Vetter, N. and Salewski, E. 1998. A comparative study of levofloxacin and ceftriaxone in the treatment of hospitalized patients with pneumonia. Scandinavian J. Infect. Dis., 30: 397404.

Ohieku, J.D., Magaji, R.A. 2013. Urinary tract infections associated with Escherichia coli: A 2005 to 2009 clinical assessment of trends in fluoroquinolones activities in Maiduguri city, Nigeria. JAPS, Vol. 3(08): pp. 084091, August, 2013.

Orenstein, R. and Wong, E.S. 1999. Urinary tract infections in adults. Am. Fam. Physician, 59(5): 1225-1234.

Prakash, D. and Saxena, R.S. 2013. Distribution and antimicrobial susceptibility pattern of bacterial pathogens causing urinary tract infection in urban community of Meerut city, India. ISRN Microbiol.,
Prats, G., Rossi, V., Salvatori, E., Mirelis, B 2006. Prulifloxacin: a new antibacterial fluoroquinolone. Expert Rev. Anti Infect. Ther., 4: 27-41.

Rani, H., Kaistha, N., Gupta, V. and Chander, J. 2011. Choice of antibiotics in community acquired UTI due to Escherichia coli in adult age group. $J$. Clin. Diag. Res., 483-485.

Razak, S.K., V. Gurushantappa. 2012. Bacteriology of Urinary Tract Infection and Antibiotic Susceptibility Pattern in a Tertiary Care Hospital in South India, IJMSPH, 1: 109-112.

Ritu Aggarwal, Uma Chaudhary, et al. 2009. Detection of Extended Spectrum $\beta$ lactamase Production among Uropathogens. J. Lab. Physicians, 1(1).

Sabir, S., Anjum, A.A., Ijaz, T., Muhammad Asad Ali, Muti-ur-Rehman Khan, Muhammad Nawaz. Isolation and antibiotic susceptibility of E. coli from urinary tract infections in a tertiary care hospital. Pak. J. Med. Sci., 30(2).

Smita Sood, Ravi Gupta. 2012. Antibiotic resistance pattern of community acquired uropathogens at a Tertiary care hospital in Jaipur, Rajasthan. JCM, 37(1).

Wimer, S.M., Schoonover, L. and Garrison, M.W. 1998. Levofloxacin: a therapeutic review. Clin. Therapeutics, 20: 104970.

\section{How to cite this article:}

Neha Mangal, Anamika Vyas, Mritunjay Kumar and Dalal, A.S. 2017. Antibiogram of Escherichia coli Isolates from Community Acquired Urinary Tract Infection: Special Reference to Fluoroquinolones Resistance. Int.J.Curr.Microbiol.App.Sci. 6(7): 22-31. doi: https://doi.org/10.20546/ijcmas.2017.607.004 